Paul D. Miller, MD, HDSc (HON)

The University of Colorado Health Sciences

Center; The Colorado Center for Bone Health, Lakewood, CO

\section{E. Michael Lewiecki, MD}

Director, New Mexico Clinical Research \& Osteoporosis Center; Director, Bone Health TeleECHO, University of New Mexico Health Sciences Center, Albuquerque, NM
Kelly Krohn, MD

The University of Arizona College of Medicine, Phoenix, AZ
Elliott Schwartz, MD

Northern California Institute for Bone Health, Inc., Orinda, CA; Department of Orthopedics, The University of California San Francisco Skeletal Health Service, San Francisco, CA

\title{
Teriparatide: Label changes and identifying patients for long-term use
}

$\mathrm{T}$ he osteoporosis agent teriparatide (Forteo) no longer carries a boxed warning about the risk of osteosarcoma, and dosing is no longer limited to 24 months of lifetime use. These labelling changes have left clinicians with the challenge of identifying patients for consideration of long-term treatment.

Teriparatide was initially approved based on only 19 months of data on fracture reduction, in contrast to the 3 years required for all other osteoporosis drugs. Given the lack of clinical trial data, in this article we offer suggestions for selecting patients for extended use of teriparatide based mostly on our extensive experience treating patients with teriparatide, some patients for longer than 2 years.

\section{CHANGES TO THE LABEL}

In November 2020, the US Food and Drug Administration (FDA) approved changes to the label for the parathyroid hormone (PTH) analogue teriparatide (PTH 1-34), by removing the 2-year lifetime treatment limitation and the boxed warning about the potential risk of osteosarcoma. ${ }^{1}$ The lifetime limitation had been established because 24 months was the longest that any woman had been treated with teriparatide in the labeling clinical trial. ${ }^{2}$

\section{Osteosarcoma boxed warning}

The deleted boxed warning (not a contraindication) regarding osteosarcoma was based on studies in Fischer 344 rats showing that high doses of teriparatide - 3 times greater than the approved human dosing based on milligrams per kilogram of body weight_-administered over most of a rat lifespan (about 24 months) were associated with the development of osteodoi:10.3949/ccjm.88a.21011 sarcoma. Based on this observation, which occurred while the clinical trial for teriparatide was ongoing, the trial was terminated early. As a result, study participants received teriparatide for an average of 19 months, with a mean observation time of 21 months. ${ }^{2}$

In the 18 years since teriparatide was approved, no increase in osteosarcoma risk has been reported in studies in animals with bone remodeling similar to that in humans (eg, monkeys). However, considering the rarity of osteosarcoma (about 1 in 250,000 adults per year), the sample size of about 60 monkeys in a study by the manufacturer of Forteo ${ }^{3}$ was too small to provide conclusive data.

In humans treated with teriparatide, there is no evidence of an increased risk of osteosarcoma. The observed incidence of osteosarcoma during a 15-year postmarketing surveillance study was no different than the background incidence rate for individuals not treated with teriparatide. ${ }^{4}$

The boxed warning has not been removed for abaloparatide (Tymlos), a synthetic analogue of PTH-related protein (PTHrP 1-34), but at the time of this writing, the FDA is in discussions with the manufacturer about removing the boxed warning. Another PTH 1-34 product (Bonsity) has also not had any recent label change.

\section{The duration of dosing}

The revised teriparatide label states that use "for more than 2 years during a patient's lifetime should only be considered if a patient remains at or has returned to having a high risk for fracture." ${ }^{1}$ Now that the teriparatide label permits use for longer than 2 years, there are practical clinical questions about the selection of patients for an extended use. 


\section{BEHIND THE ORIGINAL FDA APPROVAL}

Teriparatide is derived from splitting the biologically active 1-34 amino-acid fragment from the intact PTH 1-84 molecule. The labeling trial for teriparatide was a placebocontrolled study with the primary end point of new vertebral compression fracture (VCF) over 3 years. $^{2}$ The FDA requires evidence that pharmacologic treatment of women with postmenopausal osteoporosis reduces fracture risk over a 3-year period compared with placebo, with a favorable balance of benefits and risks.

\section{Are there osteosarcoma risks?}

When osteosarcoma was observed in the Fischer 344 rats receiving teriparatide, the study sponsor halted the human trial while the rat data were evaluated. After the FDA concluded that the osteosarcoma risk was confined to the rats, they encouraged the manufacturer to resume the clinical trial. However, by this time, approximately $50 \%$ of the patients who had been enrolled in the initial registration trial had switched to treatment with alendronate, resulting in loss of statistical power as defined by the study design. Nevertheless, a subsequent evaluation of the clinical data found that teriparatide was effective in preventing fractures and well tolerated. ${ }^{2}$

for more than

2 years during

a lifetime

should be

considered

only if a patient

is at high risk

for fracture

\section{WHY THE TIME-LIMIT CHANGE FOR DOSING?}

The FDA approved teriparatide for the treatment of postmenopausal women with osteoporosis who are at high risk for fracture, with a 24-month lifetime limit of use. High fracture risk was described as a history of osteoporotic fracture or multiple risk factors for fracture, or as failure of or inability to tolerate osteoporosis therapy. ${ }^{1}$

In 2020, 18 years after the FDA approval of teriparatide, the incidence of osteosarcoma in patients treated for 2 years was reported as being lower than the natural incidence rate of osteosarcoma in adults. ${ }^{4}$ Use of teriparatide for more than 2 years during a patient's lifetime should be considered only if a patient remains at or has returned to having a high risk for fracture. ${ }^{1}$ Determination of high risk, therefore, is a clinical decision to be made by the clinician.
The 2019 Endocrine Society clinical practice guidelines included patient profiles representing examples of high and very high fracture risk ${ }^{5}$ :

- High risk: T-score of minus 2.5 or below, or prior hip or vertebral fracture, or high fracture probability by the fracture risk assessment tool (FRAX) (10-year probability of major osteoporotic fracture $\geq 20 \%$, or 10-year probability of hip fracture $\geq 3 \%$ )

- Very high risk: T-score of minus 2.5 or below and 1 or more fractures, or multiple vertebral fractures, or severe vertebral fracture.

The Endocrine Society guidelines suggest that anabolic therapy with teriparatide or abaloparatide be considered the first-line for treatment for up to 2 years in postmenopausal women with osteoporosis who are at very high risk of fracture. ${ }^{5}$

For initial therapy in patients at high risk, there is evidence that teriparatide should be started first, followed by an antiresorptive agent (eg, bisphosphonate, estrogen, selective estrogen receptor modulator, calcitonin, denosumab) because the bone formation effects of teriparatide are blunted by initiating therapy with an antiresorptive agent. Thus, the sequence of treatment is important. ${ }^{6,7}$

Abaloparatide is a molecular modification of PTH-related protein that is synthesized with $76 \%$ homology to human PTH-related protein. This modification conveys to abaloparatide a different binding configuration to its receptor (PTH receptor type 1) than teriparatide. In clinical practice, the resulting stimulation of osteoblasts by abaloparatide results in faster and greater increases in bone mineral density (BMD) than with teriparatide. ${ }^{8}$

\section{Use for glucocorticoid-induced osteoporosis} In the teriparatide clinical trial that led to the regulatory approval of teriparatide for the treatment of glucocorticoid-induced osteoporosis, participants were randomized to receive teriparatide or alendronate for a total of 3 years. ${ }^{9}$ The primary end point was change in BMD at the lumbar spine, and vertebral fracture risk reduction was a secondary outcome measure.

Results showed that BMD increased significantly more with teriparatide than with alendronate, and new radiographic and clinical vertebral fractures were reduced to a greater extent. These findings supported the 
FDA approval of teriparatide to treat glucocorticoid-induced osteoporosis, although the recommended 24-month lifetime treatment duration was not extended.

The superior effects of teriparatide over alendronate are biologically plausible, as glucocorticoids inhibit osteoblast recruitment and activity, ${ }^{10,11}$ while teriparatide stimulates osteoblastic bone formation and alendronate inhibits it.

\section{CLINICAL IMPLICATIONS OF EXTENDED USE}

In our opinion, long-term use of teriparatide can be considered in high-risk patients receiving long-term glucocorticoid therapy. However, we still need more data on the safety and efficacy of long-term use.

\section{What about hypercalcemia?}

Hypercalcemia was seen more often in the pivotal clinical trial with teriparatide $(6.1 \%)$ than with abaloparatide (3.4\%). ${ }^{8}$ Serum calcium ideally should be drawn 16 hours or more after the injection to avoid measuring transient calcium elevations that are probably not clinically relevant. A common clinical practice protocol is to measure the serum calcium 1 month and 3 months after starting teriparatide. If no hypercalcemia appears during that time, it is very rare for hypercalcemia to appear later.

Prolonged treatment with teriparatide seems logical for patients with glucocorticoidinduced osteoporosis who cannot be managed with antiresorptive medications. Although there is a dose-response relationship with glucocorticoids and fractures, even low doses of glucocorticoids (eg, prednisone $2.5 \mathrm{mg} /$ day) are associated with elevated fracture risk compared with no glucocorticoids. ${ }^{12}$

\section{What about measuring bone quality?}

Glucocorticoids induce a decline of BMD and have adverse effects on bone quality independent of BMD. Bone quality has been described as the non-BMD properties of bone, such as architecture, turnover, damage accumulation, mineralization, and material properties that also determine bone strength..$^{13,14}$

While we can accurately measure and monitor BMD with dual-energy $\mathrm{x}$-ray absorptiometry (DXA),${ }^{15,16}$ we lack the capacity to measure bone quality in clinical practice. The trabecular bone score is a measurement derived from DXA of the lumbar spine that is a surrogate for trabecular microarchitecture, an important component of bone that is an independent predictor of fracture risk. ${ }^{17}$ The development of the trabecular bone score is a major advance in fracture risk predictability, and it is included in the FRAX algorithm to estimate the 10-year probability of fracture.

Although the trabecular bone score may improve with anabolic therapy for osteoporosis (eg, with teriparatide), it does not reliably increase with antiresorptive agents. ${ }^{18}$ Antiresorptive agents, however, may improve some aspects of bone quality, but this effect has not been systematically validated in human beings. ${ }^{19}$

While the capacity to measure bone quality in clinical practice is limited, there are measurements, used mostly in research settings, that may be helpful. Transiliac nondecalcified double tetracycline-labeled bone biopsy provides quantitative bone histomorphometry, ${ }^{20}$ and high-resolution peripheral quantitative computed tomography measures bone trabecular structure at peripheral skeletal sites with a resolution of $82 \mu \mathrm{m} .{ }^{21}$ The tomography test, however, is a research tool that is not currently applicable to clinical practice. Neither of these methodologies is widely available.

\section{Which patients are most likely to benefit from long-term teriparatide use?}

When considering the clinical benefit of continuing teriparatide beyond 2 years, there are no published studies addressing this issue (notwithstanding the 3-year data with teriparatide for glucocorticoid-induced osteoporosis ${ }^{9}$ ). Interestingly, a review of clinical data on teriparatide treatment over time showed that while BMD declined rapidly after discontinuing teriparatide, fracture rates did not increase for as long as 18 months after teriparatide discontinuation..$^{22}$ There is no definitive explanation for this observation, although it suggests that improvements in bone quality with teriparatide persist longer than the increases in BMD.

In our opinion, there are clinical features that identify patients who may benefit from long-term administration of teriparatide (Table 1). Current glucocorticoid users are at high risk for fractures and remain at high or very high risk as long as they use glucocorticoids. Additionally, patients at high risk for fracture who
Long-term use of teriparatide can be considered in high-risk patients on long-term glucocorticoid therapy, but more data on safety and efficacy are needed 


\section{TABLE 1}

\section{Features of patients who may benefit from long-term teriparatide use}

Very high fracture risk, unable to come off glucocorticoid therapy

High fracture risk, with P1NP level that remains high after 2 years on teriparatide

High fracture risk, with multiple vertebral compression fractures at baseline but none while on teriparatide

Adynamic renal bone disease

Severe chronic obstructive pulmonary disease and vertebral compression fractures

P1NP = procollagen type $1 \mathrm{~N}$-terminal propeptide

have a level of the bone-formation marker procollagen type $1 \mathrm{~N}$-terminal propeptide (P1NP) that remains above the upper limit of the reference range after 2 years of treatment with teriparatide should continue therapy because the elevated P1NP indicates that new bone formation is continuing. An increase of P1NP

Patients at high or very high fracture risk who have severe COPD and vertebral compression fractures may be candidates for long-term teriparatide therapy of more than $10 \mu \mathrm{g} / \mathrm{L}$ from baseline while on teriparatide therapy is correlated with improvements in BMD and bone strength. ${ }^{23}$

As with all osteoporosis medications, the $\mathrm{BMD}$ or the bone turnover marker may not always change over time while the patient is on therapy. However, because a stable BMD may be an acceptable response and the P1NP may not increase, we feel that as long as the patient does not suffer a fracture, treatment should be continued. Furthermore, patients at high or very high fracture risk who have multiple VCFs at baseline but no incidence of VCF while on teriparatide may be candidates for treatment longer than 2 years, especially if a bone formation marker such as P1NP is still above the reference range.

Data show that VCFs are associated with a high risk of more VCFs and non-VCFs in untreated patients ${ }^{24}$ and a high 10 -year mortality. ${ }^{25}$ In its labeling clinical trial, ${ }^{2}$ teriparatide reduced VCF incidents by about $80 \%$ over 19 months, similar to the VCF risk reduction with abaloparatide. ${ }^{8}$

Renal-associated adynamic bone disease.
There are reports suggesting that patients at high or very high fracture risk who have adynamic renal bone disease may respond to treatment with teriparatide. ${ }^{26-29}$ Idiopathic adynamic renal bone disease is a form of renal bone disease characterized on bone biopsy as very low bone turnover, very low bone formation, and poor osteoid development with an increased risk for low-trauma fractures. ${ }^{26,27}$

Many patients who have stage 4 or 5 or 5D chronic kidney disease (especially with diabetic renal disease), who have bone-biopsy-documented adynamic renal bone disease have serum PTH levels below 100 to $150 \mathrm{pg} /$ $\mathrm{mL}$, and who have a bone-specific alkaline phosphatase in the lower quartile of the reference range have a high positive predictive value for having adynamic bone disease. ${ }^{28} \mathrm{Al}$ though there have been a few case reports of teriparatide improving bone formation parameters measured by paired bone biopsies in this patient population, ${ }^{30,31}$ data are needed from prospective clinical trials or large observational trials to validate its long-term efficacy.

Severe chronic obstructive pulmonary disease (COPD) and VCFs. Finally, patients at high risk or very high risk of fracture who have severe COPD and VCFs may be candidates for long-term use of teriparatide. $\mathrm{Pa}$ tients with COPD and VCFs are at high risk for more fractures and have increased mortality risk. ${ }^{25}$ For each VCF in these patients, there is a loss of about $8 \%$ of vital capacity. ${ }^{32-34}$ These patients cannot afford any additional loss of lung function.

Long-term use of teriparatide seems justified in these patients to reduce mortality risk by preventing more VCFs. In our opinion, teriparatide or abaloparatide should be the initial therapy in these patients, given that anabolic agents reduce the incidence of VCF to a greater extent than bisphosphonates. ${ }^{35}$

\section{CONTINUING CHALLENGES}

With the labeling changes to teriparatide, clinicians face the challenge of identifying patients for longer treatment. Although the evidence for guiding such a decision is limited, we have suggested clinical circumstances in which longterm teriparatide may be appropriate.

It is unclear how long to continue teripa- 
ratide beyond 2 years. We suggest that practitioners consider continuing treatment as long as P1NP levels remain appropriately elevated and the patient has not had new VCFs.

Finally, we encourage the development and implementation of clinical investigations to explore the potential additional benefits of

\section{REFERENCES}

1. Forteo [package insert]. Indianapolis, IN: Eli Lilly and Co; 2020. https:/Mwww.accessdata.fda.gov/drugsatfda_docs/abe/2020/021318Orig15054/bl.pdf. Accessed Augus $16,2021$.

2. Neer RM, Arnaud CD, Zanchetta JR, et al. Effect of parathyroid hormone (1-34) on fractures and bone mineral density in postmenopausal women with osteoporosis. N Engl J Med 2001; 344(19):1434-1441. doi:10.1056/NEJM200105103441904

3. Vahle JL, Zuehlke U, Schmidt A, Westmore M, Chen P, Sato M. Lack of bone neoplasms and persistence of bone efficacy in cynomolgus macaques after long-term treatment with teriparatide [rhPTH(1-34)]. J Bone Miner Res 2008; 23(12):20332039. doi:10.1359/jbmr.080807

4. Gilsenan A, Midkiff K, Harris D, Kellier-Steele N, McSorley D, Andrews EB. Teriparatide did not increase adult osteosarcoma incidence in a 15-year us postmarketing surveillance study. J Bone Miner Res 2021; 36(2):244-251. doi:10.1002/jbmr.4188

5. Eastell R, Rosen C, Black DM, Cheung AM, Murad MH, Shoback D. Pharmacological management of osteoporosis in postmenopausal women: an Endocrine Society Clinical Practice Guideline. J Clin Endocrinol Metab 2019; 104(5):1595-1622. doi:10.1210/jc.2019-00221

6. Tsai JN, Uihlein AV, Lee $\mathrm{H}$, et al. Teriparatide and denosumab, alone or combined, in women with postmenopausal osteoporosis: the DATA study randomised trial. Lancet 2013; 382(9886):50-56. doi:10.1016/50140-6736(13)60856-9

7. Cosman F, Nieves JW, Dempster DW. Treatment sequence matters: anabolic and antiresorptive therapy for osteoporosis. J Bone Miner Res 2017; 32(2):198-202. doi:10.1002/jbmr.3051

8. Miller PD, Hattersley G, Riis BJ, et al. Effect of abaloparatide vs placebo on new vertebral fractures in postmenopausal women with osteoporosis: a randomized clinical trial. JAMA 2016; 316(7):722-733. doi:10.1001/jama.2016.11136

9. Saag KG, Zanchetta JR, Devogelaer JP, et al. Effects of teriparatide versus alendronate for treating glucocorticoid-induced osteoporosis: thirty-six-month results of a randomized, double-blind, controlled trial. Arthritis Rheum 2009; 60(11):33463355. doi:10.1002/art.24879

10. Canalis E. The fate of circulating osteoblasts. N Engl J Med 2005; 352(19):20142016. doi:10.1056/NEJMe058080

11. Baron R, Gori F. Targeting WNT signaling in the treatment of osteoporosis. Curr Opin Pharmacol 2018; 40:134-141. doi:10.1016/j.coph.2018.04.011

12. van Staa TP, Leufkens HG, Cooper C. The epidemiology of corticosteroid-induced osteoporosis: a meta-analysis. Osteoporos Int 2002; 13(10):777-787. doi:10.1007/5001980200108

13. Seeman E, Delmas PD. Bone quality - the material and structural basis of bone strength and fragility. N Engl J Med 2006; 354(21):2250-2261. doi:10.1056/NEJMra053077

14. Licata A. Bone density vs bone quality: what's a clinician to do? Cleve Clin J Med 2009; 76(6):331-336. doi:10.3949/ccjm.76a.08041

15. Miller PD, Zapalowski C, Kulak CA, Bilezikian JP. Bone densitometry: the best way to detect osteoporosis and to monitor therapy. J Clin Endocrinol Metab 1999; 84(6):1867-1871. doi:10.1210/jcem.84.6.5710

16. Kendler DL, Compston J, Carey JJ, Wu CH, Ibrahim A, Lewiecki EM. Repeating measurement of bone mineral density when monitoring with dual-energy $\mathrm{x}$-ray absorptiometry: 2019 ISCD official position. J Clin Densitom 2019; 22(4):489-500. doi:10.1016/j.jocd.2019.07.010

17. Hans D, Štenová E, Lamy O. The trabecular bone score (TBS) complements DXA and the FRAX as a fracture risk assessment tool in routine clinical practice. Curr Osteoporos Rep 2017; 15(6):521-531. doi:10.1007/s11914-017-0410-z

18. Krohn K, Schwart EN, Chung YS, Lewiecki EM. Dual-energy x-ray absorptiometry monitoring with trabecular bone score: 2019 ISCD official position. J Clin Densitom 2019; 22(4):501-505. doi:10.1016/.jocd.2019.07.006

19. Borah B, Dufresne TE, Chmielewski PA, Johnson TD, Chines A, Manhart MD. Rise- onger-term use of teriparatide and other analic agents.

\section{DISCLOSURES}

tz has disclosed consulting, membership on advisory committe we panels, and teaching and speaking for Amgen; and consulting, arch/independent contracting, membership on advisory committee or iew panels, and teaching and speaking for Radius Health. The other au作 ributions, could be perceived as a potential conflict of interest.

dronate preserves bone architecture in postmenopausal women with osteoporosis as measured by three-dimensional microcomputed tomography. Bone 2004; 34(4):736-746. doi:10.1016/j.bone.2003.12.013

20. Dempster DW, Compston JE, Drezner MK, et al. Standardized nomenclature, symbols, and units for bone histomorphometry: a 2012 update of the report of the ASBMR Histomorphometry Nomenclature Committee. J Bone Miner Res 2013, 28(1):2-17. doi:10.1002/jbmr.1805

21. Engelke $K$, Libanati C, Fuerst T, Zysset P, Genant HK. Advanced CT based in vivo methods for the assessment of bone density, structure, and strength. Curr Osteoporos Rep 2013; 11(3):246-255. doi:10.1007/s11914-013-0147-2

22. Lindsay R, Scheele WH, Neer R. Sustained vertebral fracture risk reduction after withdrawal of teriparatide in postmenopausal women with osteoporosis. Arch Intern Med 2004; 164(18):2024-2030. doi:10.1001/archinte.164.18.2024

23. Krege JH, Lane NE, Harris JM, Miller PD. PINP as a biological response marker during teriparatide treatment for osteoporosis. Osteoporos Int 2014; 25(9):2159-2171. doi:10.1007/s00198-014-2646-0

24. Kendler DL, Bauer DC, Davison KS, et al. Vertebral fractures: clinical importance and management. Am J Med 2016; 129(2):221.e1-e10. doi:10.1016/j.amjmed.2015.09.020

25. Bliuc D, Nguyen ND, Milch VE, Nguyen TV, Eisman JA, Center JR. Mortality risk associated with low-trauma osteoporotic fracture and subsequent fracture in men and women. JAMA 2009; 301(5):513-521. doi:10.1001/jama.2009.50

26. Massy Z, Drueke T. Adynamic bone disease is a predominant bone pattern in early stages of chronic kidney disease. J Nephrol 2017; 30(5):629-634. doi:10.1007/s40620-017-0397-7

27. Cannata Andía JB. Adynamic bone and chronic renal failure: an overview. Am J Med Sci 2000; 320(2):81-84. doi:10.1097/00000441-200008000-00003

28. Garrett G, Sardiwal S, Lamb EJ, Goldsmith DJ. PTH—a particularly tricky hormone: why measure it at all in kidney patients?. Clin J Am Soc Nephrol 2013; 8(2):299-312. doi:10.2215/CNN.09580911

29. Couttenye MM, D’Haese PC, Deng JT, Van Hoof VO, Verpooten GA, De Broe ME. High prevalence of adynamic bone disease diagnosed by biochemical markers in a wide sample of the European CAPD population. Nephrol Dial Transplant 1997; 12(10):2144-2150. doi:10.1093/ndt/12.10.2144

30. Peugh J, Khalil A, Chan MR, Hansen KE. Teriparatide treatment for hypercalcemia associated with adynamic bone disease. JBMR Plus 2019; 3(7):e10176. doi:10.1002/jbm4.10176

31. Palcu P, Dion N, Ste-Marie LG, et al. Teriparatide and bone turnover and formation in a hemodialysis patient with low-turnover bone disease: a case report. Am J Kidney Dis 2015; 65(6):933-936. doi:10.1053/.ajkd.2015.01.025

32. Chen CM, Lin PY, Chen YC, et al. Effects of teriparatide on lung function and pain relief in women with multiple osteoporotic vertebral compression fractures. Surg Neurol Int 2014; 5(suppl 7):S339-S342. doi:10.4103/2152-7806.139653

33. Lehouck A, Boonen S, Decramer M, Janssens W. COPD, bone metabolism, and osteoporosis. Chest 2011; 139(3):648-657. doi:10.1378/chest.10-1427

34. Leech JA, Dulberg C, Kellie S, Pattee L, Gay J. Relationship of lung function to severity of osteoporosis in women. Am Rev Respir Dis 1990; 141(1):68-71. doi:10.1164/ajrccm/141.1.68

35. Kendler DL, Marin F, Zerbini CAF, et al. Effects of teriparatide and risedronate on new fractures in post-menopausal women with severe osteoporosis (VERO): a multicentre, double-blind, double-dummy, randomised controlled trial. Lancet 2018; 391(10117):230-240. doi:10.1016/50140-6736(17)32137-2

Address: Paul D. Miller, MD, HDSc (HON), Colorado Center for Bone Health, PC, 6655 W. Jewell Avenue, Suite \#2, Lakewood, CO 80232; pmiller@millerbonecenter.com 